УДК 621.315 .592

\title{
Разогрев электронов в чистом Ge в квантовом магнитном поле при термическом возбуждении носителей заряда
}

\author{
(C) В.Ф. Банная ${ }^{1}$, E.В. Никитина ${ }^{2, \uparrow}$ \\ ${ }^{1}$ Московский педагогический государственный университет, \\ 119991 Москва, Россия \\ ${ }^{2}$ Российский университет дружбы народов, \\ 117198 Москва, Россия \\ E-mail: enikitina@sci.pfu.edu.ru
}

Поступила в Редакцию 8 октября 2019 г.

В окончательной редакции 15 октября 2019 г.

Принята к публикации 15 октября 2019 г.

Рассмотрены результаты экспериментального исследования разогрева носителей заряда электрическим полем $(\mathbf{E})$ в чистом $\mathrm{Ge}$ в квантовом магнитном поле $(\mathbf{H})$ при $(\mathbf{E} \perp \mathbf{H})$ при низких температурах, $T=4.2,1.8 \mathrm{~K}$, в условиях термовозбуждения. Показано, что в этих условиях на среднее время жизни носителей влияет зависимость от $E$ и $H$ коэффициента термической ионизации. Полученные результаты качественно согласуются с теорией каскадного захвата носителей на изолированные центры в скрещенных электрическом и магнитном полях.

Ключевые слова: квантованность, каскадный захват, электрическое поле, постоянная Холла, магнитное поле.

DOI: 10.21883/FTP.2020.03.49021.9282

\section{1. Введение}

В работе [1] было рассмотрено влияние квантового магнитного поля на разогрев носителей заряда при температуре $T=4.2 \mathrm{~K}$ в условиях фотовозбуждения носителей. Как известно, в этом случае зависимость концентрации свободных электронов $n(E, H)$, где $E$ и $H$ - напряженности электрического и магнитного полей, определяется поведением среднего времени жизни $\left\langle\tau_{c}\right\rangle$ носителей заряда. Однако при термогенерации характер зависимостей $n(E, H)$ при $T=4.2 \mathrm{~K}$ определяется также полевыми зависимостями коэффициента термической ионизации $A_{T}(E, H)$. Поэтому представляет интерес изучение полевых зависимостей коэффициента Холла $R_{\mathrm{H}}(E, H)$ и $\left\langle\tau_{c}\right\rangle(H)$ при низких температурах в условиях термического возбуждения.

\section{2. Условия и результаты эксперимента}

Измерения выполнялись при $T=4.2$ и $1.8 \mathrm{~K}$. Свободные носители в зоне создавались только термовозбуждением, фоновая подсветка от верхней части криостата был перекрыта специальными холодными металлическими фильтрами. Остальные условия эксперимента и параметры образцов $n$ - и $p$-Ge были такие же, как и в работе [1].

Измеряемыми величинами являлись коэффициент Холла $R_{\mathrm{H}}$ и удельная электропроводность $\sigma$. Электрическое поле варьировалось от $\sim 10^{-1}$ до $\sim 50 \mathrm{~B} / \mathrm{cM}$, магнитное поле - от 0 до $\sim 5 \cdot 10^{4}$ Э.
На рис. 1 представлены зависимости $R_{\mathrm{H}}(E)$ при разных $H$ для двух образцов $n$-Ge (параметры образцов приведены в подписи к рисунку).

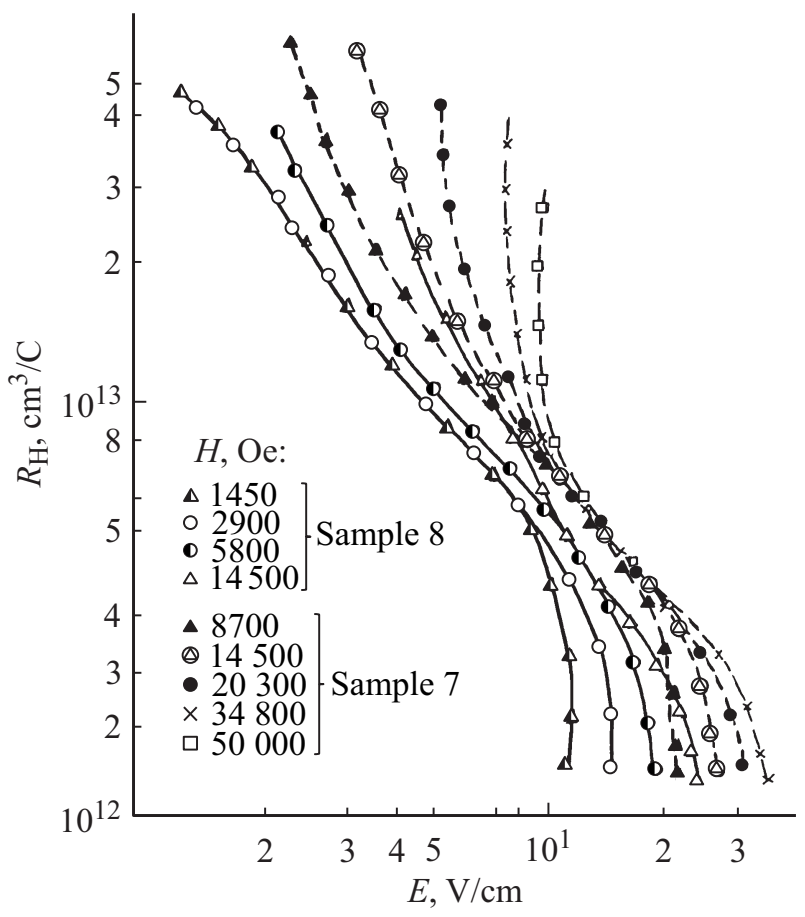

Рис. 1. Зависимости $R_{\mathrm{H}}(E)$ в образцах $n$-Ge при разных $H$ в условиях термовозбуждения при $T=4.2 \mathrm{~K}$. Образец 8: $n$-Ge, $N_{d}=7 \cdot 10^{13} \mathrm{~cm}^{-3}, N_{a}=6 \cdot 10^{12} \mathrm{~cm}^{-3}, K=8.6 \%$. Образец 7: $n$-Ge $N_{d}=1.7 \cdot 10^{13} \mathrm{~cm}^{-3}, N_{a}=2.7 \cdot 10^{12} \mathrm{~cm}^{-3}, K=14 \%$. $K-$ степень компенсации примесей. 
Условия квантования $\hbar \omega_{c}>k_{0} T\left(\omega_{c}-\right.$ циклотронная частота, $k_{0}$ - постоянная Больцмана) при $T=4.2 \mathrm{~K}$ выполняются в $n$-Ge начиная с $H=H_{q n} \gtrsim 5$ кЭ, в $p$-Ge для тяжелых дырок - начиная с $H=H_{q n} \gtrsim 9$ кЭ $\left(H_{q n}-\right.$ напряженность квантующего магнитного поля).

Из рис. 1 видно, что при $H<H_{q n} R_{\mathrm{H}}$ не зависит от $H$ вплоть до $E=E_{\mathrm{br}}\left(E_{\mathrm{br}}\right.$ - поле электрического пробоя). При $H \gtrsim H_{q n}$ наблюдается резкое увеличение коэффициента Холла с увеличением $H$. (К сожалению, из-за очень большой величины сопротивления образцов в магнитном поле провести измерения в области малых электрических полей не удается). С ростом $E$ коэффициент Холла падает, причем тем сильнее, чем больше значение $H$.

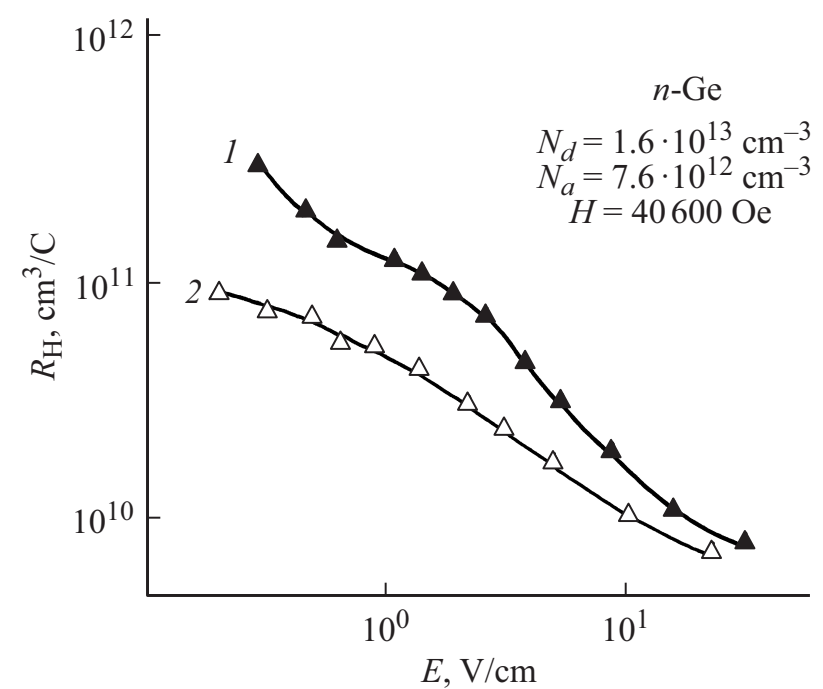

Рис. 2. Зависимости $R_{\mathrm{H}}(E)$ для $n$-Ge в квантовом магнитном поле при температурах $T, \mathrm{~K}: 1-1.8,2-4.2$.

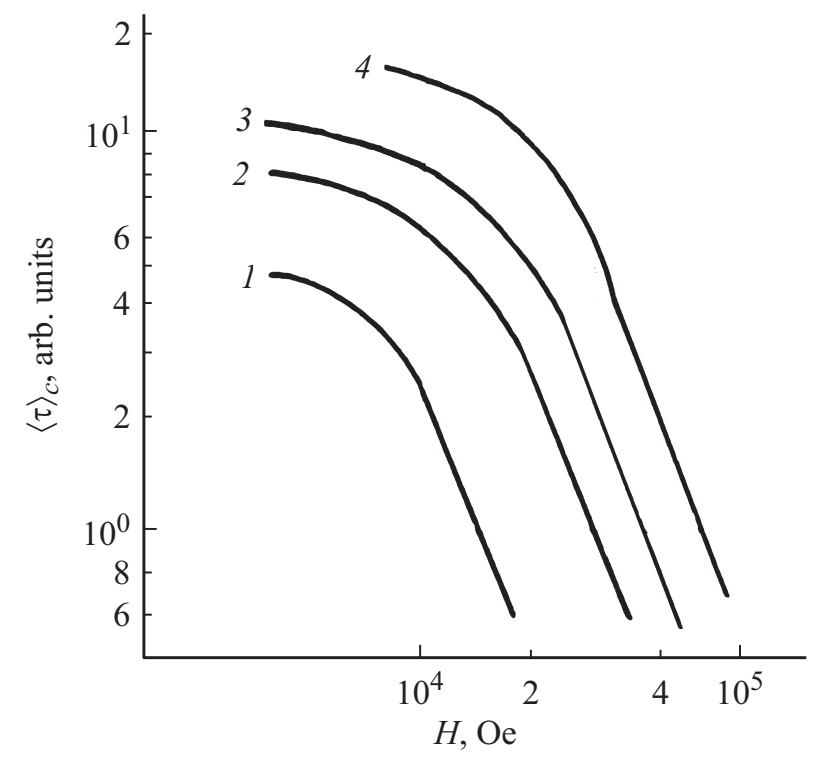

Рис. 3. Зависимости $\left\langle\tau_{c}\right\rangle(H)$ для $n$-Ge (образец 7, см. рис. 1) в условиях термовозбуждения при разных значениях напряженности электрического поля $E, \mathrm{~B} / \mathrm{cm:} 1-3,2-5,3-6$, $4-8$.
На рис. 2 приведены зависимости $R_{\mathrm{H}}(H)$ для образца $n$-Ge при $T=4.2$ и $1.8 \mathrm{~K}$.

Из рис. 1 и 2 видно, что характер приведенных кривых такой же, как и для аналогичных образцов в условиях фотовозбуждения [1], но в случае термовозбуждения зависимость значительно сильнее. Так, например, в случае фотовозбуждения $\left\langle\tau_{c}\right\rangle \propto H^{-1}$, в случае термовозбуждения (рис. 3) $\left\langle\tau_{c}\right\rangle \propto H^{-3}$; аналогично и для зависимостей от $E$ : при фотовозбуждении и $H=40600 Э\left\langle\tau_{c}\right\rangle \propto E^{2}$, в темновых условиях (рис. 3) $\left\langle\tau_{c}\right\rangle \propto E^{(6-7)}$. С ростом электрического поля эта зависимость ослабляется, хотя и остается при термическом возбуждении сильнее, чем при фотовозбуждении. Так, при $E \approx 10 \mathrm{~B} /$ см при наличии подсветки [1] $R_{\mathrm{H}} \propto E^{-0.5}$, в темновых условиях (рис. 1) $R_{\mathrm{H}} \propto E^{-1}$. В этом интервале электрических полей в обоих случаях $R_{\mathrm{H}}$ (следовательно, и $\left\langle\tau_{c}\right\rangle$ ) практически не зависит от $H$ (рис. 3). Дальнейшая зависимость $R_{\mathrm{H}}(H)$ наблюдается лишь при $E \sim E_{\mathrm{br}}$.

Понижение температуры (см. рис. 2) усиливает зависимость $R_{\mathrm{H}}$ от $E$.

\section{3. Обсуждение экспериментальных результатов}

Для объяснения полученных результатов обратимся к теории каскадного захвата носителей заряда на изолированный примесный центр в квантовых магнитных полях [2,3]. При таком захвате происходит испускание фонона, величина энергии которого в квантующих магнитных полях $\sim \sqrt{\hbar \omega_{c} m s^{2}}$, что намного больше энергии соответствующего фонона в отсутствие поля $\sqrt{k_{0} T m s^{2}}$ $\left(\hbar \omega_{c} \gg k_{0} T\right)$.

Как показано в $[2,3]$, в случае термического возбуждения носителей концентрация их в зоне равна

$$
n(E)=\frac{N_{d}-N_{a}}{N_{d}\left(k_{0} T_{e}\right)} \frac{\varphi^{*}}{2 \sqrt{2}}\left(\frac{m k_{0} T_{e}^{*}}{\pi \hbar^{2}}\right)^{3 / 2} \exp \left\{-\left[\frac{\varepsilon_{i}-\varepsilon_{E}}{k_{0} T}\right]\right\},
$$

где $N_{d}, N_{a}$ - концентрации донорных и акцепторных примесей соответственно, $\varphi^{*}-$ величина деформационного потенциала в квантовом магнитном поле, $T_{e}-$ эффективная температура электронов в электрическом поле, $T_{e}^{*}$ - эффективная температура электронов в квантовом магнитном поле, $m$ - эффективная масса носителя заряда, $\varepsilon_{i}$ - энергия ионизации примесного центра, $\varepsilon_{E}$ - энергия деформации кулоновского центра захвата электрическим полем, $\sqrt{\hbar \omega_{c} m s^{2}}-$ энергия фонона в квантующих магнитных полях, испускаемого носителем заряда при его каскадном захвате на изолированный притягивающий центр, $\sqrt{k_{0} T m s^{2}}-$ энергия фонона в отсутствие $H$.

Величины $\varphi^{*}$ и $k_{0} T_{e}^{*}$ зависят от конкретных условий эксперимента.

Так, в случае скрещенных сильного $H$ и греющего $E$, если $k_{0} T \gg 8 m s^{2} k_{0} T_{e}^{*} / k_{0} T$, то $\varphi^{*}=3^{-3 / 2}$, $k_{0} T_{e}^{*}=\left(e E / m s \omega_{c}\right)^{2} k_{0} T$, т. е. $n \propto E^{3}$ и $n \propto H^{-3}$; если 
$k_{0} T \approx 8 m s^{2} k_{0} T_{e}^{*} / k_{0} T$, то $\varphi^{*}=1 / 27 \sqrt{\pi}, k_{0} T_{e}^{*}=(32 / 25)$ $\times\left(e E / m s \omega_{c}\right)^{4} m s^{2}$ и $n \propto E^{6}, n \propto H^{-6}$.

В случае квантового предела $\sqrt{\hbar \omega_{c} m s^{2}} \gg k_{0} T$

$$
n=\frac{N_{d}-N_{a}}{N}\left(\frac{m}{2 \pi}\right)^{3 / 2} \frac{\omega_{c}\left(k_{0} T\right)^{1 / 2}}{\hbar^{2}} e^{-\varepsilon_{i} / k_{0} T},
$$

но при выполнении условия $\hbar \omega_{c} \ll \varepsilon_{i} n \propto H$.

В скрещенных электрическом и квантовом магнитном полях при $k_{0} T_{e}^{*} \gg \hbar \omega_{c}$ (т.е. квантованием энергии в зоне можно принебречь) зависимость $\left\langle\tau_{c}\right\rangle(E, H)$ усиливается:

$$
\left\langle\tau_{c}\right\rangle(E, H)=\frac{A \varphi^{*}}{\sqrt{2}}\left(\frac{m k_{0} T_{e}^{*}}{\pi \hbar^{2}}\right)^{3 / 2}\left\langle\tau_{c}\right\rangle(H) .
$$

Подставляя значения $A, \varphi^{*}, \quad k_{0} T_{e}^{*}\left\langle\tau_{c}\right\rangle \quad$ (см. $\left.\quad[1-3]\right)$ для случая вынужденного испускания фононов, имеем $\left\langle\tau_{c}\right\rangle \propto E^{3} / H^{5}$, для случая спонтанного испускания фононов $\left\langle\tau_{c}\right\rangle \propto E^{6} / H^{9}$.

Сопоставляя экспериментальные данные с расчетом $[2,3]$, можно говорить только о качественном соответствии в связи с тем, что для Ge строго не реализуются те экспериментальные условия, для которых выполнен расчет; кроме того, для $p$-Ge существенный вклад при низких температурах в сильных электрических полях вносит рассеяние на оптических фононах, которое в $[2,3]$ не учитывалось.

\section{4. Заключение}

Таким образом, в работе рассмотрены особенности разогрева носителей заряда в чистом $\mathrm{Ge}$ $\left(N_{a}+N_{d} \lesssim 10^{13} \mathrm{~cm}^{-3}\right)$ электрическим полем в квантующих магнитных полях в условиях термогенерации носителей при низких температурах $(T=4.2,1.8 \mathrm{~K})$.

Получены следующие результаты.

- На поведении $\left\langle\tau_{c}\right\rangle(E, H)$ сказывается зависимость от электрического и магнитного полей коэффициента термической ионизации; характер кривых $\left\langle\tau_{c}\right\rangle(H, E)$ при $H \geq H_{q n}$ такой же, как и при фотовозбуждении, т.е. с ростом $H$ величина $\left\langle\tau_{c}\right\rangle$ падает, а с ростом $E$ растет, однако количественно все зависимости более сильные.

- Понижение температуры приводит к еще большему усилению указанных процессов.

- Наблюдаемые в эксперименте зависимости $\left\langle\tau_{c}\right\rangle(H, E)$ можно аппроксимировать степенными функциями $\left\langle\tau_{c}\right\rangle \propto H^{-3},\left\langle\tau_{c}\right\rangle \propto E^{(6-7)}$.

- Полученные результаты качественно согласуются с теорией захвата носителей заряда в скрещенных электрическом и магнитном полях, развитой в работах $[2,3]$.

\section{Конфликт интересов}

Авторы заявляют, что у них нет конфликта интересов.

\section{Список литературы}

[1] В.Ф. Банная, Е.В. Никитина. ФТП, 53 (1), 13 (2019).

[2] В.Н. Абакумов, Л.Н. Крещук, И.Н. Яссиевич. ФТП, 12 (2), 264 (1978).

[3] Л.Н. Крещук. ФТП, 13, 919 (1979).

Редактор Л.В. Шаронова

\section{Heating of electrons in pure Ge in quantum magnetic field at thermal excitation of charge carriers}

\author{
V.F. Bannaya ${ }^{1}$, E.V. Nikitina ${ }^{2}$ \\ ${ }^{1}$ Moscow State University of Education, \\ 119991 Moscow, Russia \\ 2Peoples' Friendship University of Russia \\ (RUDN University), \\ 117198 Moscow, Russia
}

\begin{abstract}
The results of an experimental study on charge carriers heating by an electric field $(\mathbf{E})$ in pure Ge in a quantum magnetic field $(\mathbf{H})$ at $(\mathbf{E} \perp \mathbf{H})$ at low temperatures $(T=4.2,1.8 \mathrm{~K})$ under thermal excitation are considered. It is shown that the dependence of thermal ionization coefficient on $E$ and $H$ affects the average carrier lifetime under these conditions. The obtained results are in qualitative agreement with the theory of cascade capture of carriers by isolated centers in crossed electric and magnetic fields.
\end{abstract}

